\title{
BER Performance of MPSK and MQAM in 2x2 Almouti MIMO Systems
}

\author{
A. S. Mindaudu ${ }^{1}$ and A. M. Miyim² \\ ${ }^{1}$ Department of Electrical Engineering, Sokoto State Polytechnic, Nigeria. \\ amindaudu@yahoo.com \\ ${ }^{2}$ Department of Electrical, Electronics \& Systems Engineering, Faculty of Engineering, \\ Universiti Kebangsaan Malaysia \\ ammiyimeeng .ukm.my
}

\begin{abstract}
Almouti published the error performance of the $2 x 2$ space-time transmit diversity scheme using BPSK. One of the key techniques employed for correcting such errors is the Quadrature amplitude modulation (QAM) because of its efficiency in power and bandwidth. In this paper we explore the error performance of the $2 \times 2$ MIMO system using the Almouti space-time codes for higher order PSK and M-ary QAM. MATLAB was used to simulate the system; assuming slow fading Rayleigh channel and additive white Gaussian noise. The simulated performance curves were compared and evaluated with theoretical curves obtained using BER tool on the MATLAB by setting parameters for random generators. The results shows that the technique used do find a place in correcting error rates of QAM system of higher modulation schemes. The model can equally be used not only for the criteria of adaptive modulation but for a platform to design other modulation systems as well.
\end{abstract}

\section{KEYWORDS}

MIMO; M-ary QAM; M-ary PSK; Eb/No; BER; STBC

\section{INTRODUCTION}

The increasing demand for modern communication systems to provide high speed multimedia wireless services places very stringent requirements on the systems. The communication systems process message symbols in digital manner with the sole objective of transmitting the symbols and recovering them correctly at the receiving end. This is not entirely an easy process especially in wireless systems where several factors come into play. For instance, limitations in bandwidth, propagation loss, time variance, noise, interference, and multipath fading, among other factors, make the wireless channel a narrow pipe through which data transmission is not very easy [1].

Wireless systems based on the MIMO technique are employed in applications that require high data rates. In such systems due to the additional antennas and signal processing chains, power problems are attendant issues to be resolved. Consequently, choice of modulation schemes and low power consumption amplifiers are critical in the MIMO systems. This is in addition to the limitation of the antenna size especially for mobile hand held devices. Coding methods and the complexity of the receivers are equally important as they impact directly on the realisation of the expected benefits of the MIMO technique in a commercial manner. 
International Journal of Information Sciences and Techniques (IJIST) Vol.2, No.5, September 2012

MIMO has become an interesting area of research simply as a result of its potential to give many orders of magnitude improvement in wireless communication performance at no cost of extra spectrum (only hardware and complexity are added) [2].

\section{THE MIMO SIGNAL MODEL}

In a MIMO system where there are $\mathrm{M}$ transmit and $\mathrm{N}$ receive antennas as shown in fig. 1 , the transmitted data signals pass through multiple paths to get to the receiving antennas. Though not shown on the diagram, but there is also noise that interferes with the data signals along the paths.

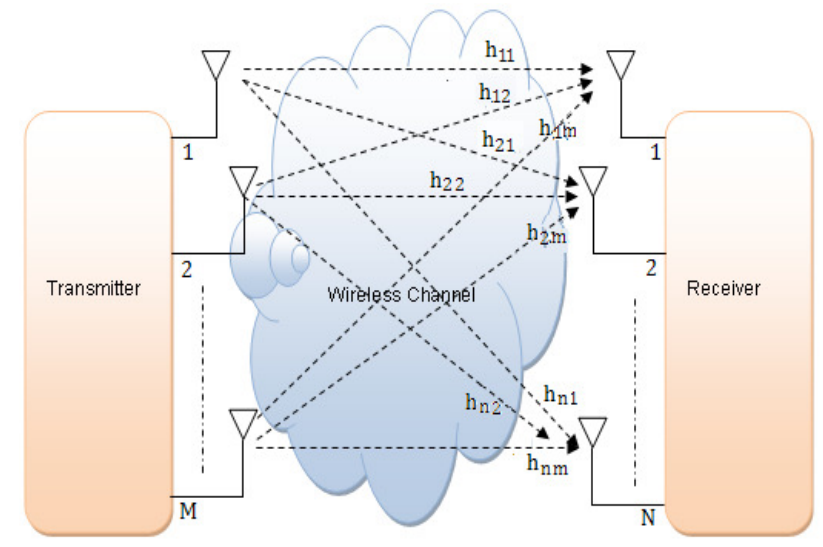

Figure 1 Basic M-transmit by N-receive MIMO system

If the channel matrix is $\mathrm{H}$, then the MIMO signal model is defined in matrix form by [2] as

$$
r=H . s+n
$$

where

$r$ is the received signal vector

$\mathrm{s}$ is the transmitted signal vector

$\mathrm{n}$ is the additive white Gaussian noise vector with zero mean and a variance of $\sigma^{2}$.

Equation 1 can also be presented as a system of linear equations given by:-

$$
\left[\begin{array}{c}
n_{1} \\
r_{2} \\
\vdots \\
\vdots \\
r_{N}
\end{array}\right]=\left[\begin{array}{cccc}
h_{11} & h_{12} & \ldots & h_{1 M} \\
h_{21} & h_{22} & \ldots & h_{2 M} \\
\vdots & \vdots & \ddots & \vdots \\
\vdots & \vdots & \ddots & \vdots \\
h_{N 1} & \ldots & \ldots & h_{N M}
\end{array}\right]\left[\begin{array}{c}
s_{1} \\
s_{2} \\
\vdots \\
\vdots \\
s_{M}
\end{array}\right]+\left[\begin{array}{c}
n_{1} \\
n_{2} \\
\vdots \\
\vdots \\
n_{N}
\end{array}\right]
$$

For a 2x2 MIMO system, the expression reduces to

$$
\left[\begin{array}{l}
n_{1} \\
n_{2}
\end{array}\right]=\left[\begin{array}{ll}
h_{11} & h_{12} \\
h_{21} & h_{22}
\end{array}\right] \cdot\left[\begin{array}{l}
s_{1} \\
s_{2}
\end{array}\right]+\left[\begin{array}{l}
n_{1} \\
n_{2}
\end{array}\right]
$$

\section{THE ALMOUTI 2X2 MIMO SCHEME}

The encoding and transmission sequence for the two transmit antennas in the Almouti scheme is shown in Table 1 [3]. 
International Journal of Information Sciences and Techniques (IJIST) Vol.2, No.5, September 2012

Table 1 - Encoding and transmission sequence for the Almouti two transmit antennas.

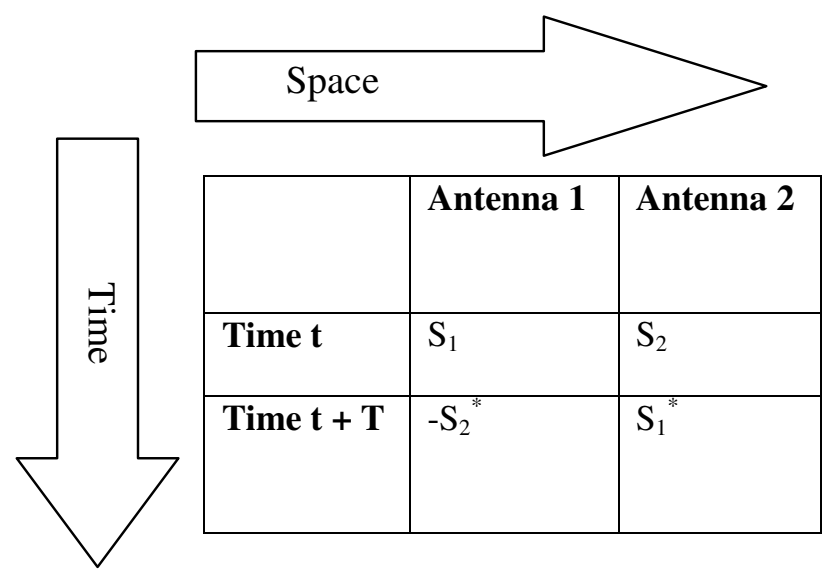

At time $t, S_{1}$ and $S_{2}$ are transmitted simultaneously by antennas 1 and 2 respectively. In the next time instant, $\mathrm{t}+\mathrm{T}$ where $\mathrm{T}$ is the symbol duration $-\mathrm{S}_{2}{ }^{*}$ and $\mathrm{S}_{1}{ }^{*}$ are transmitted simultaneously by antennas 1 and 2 respectively, where * denotes complex conjugation.

A generalised frame design for the scheme requires a concatenation of $2 \times 2$ blocks of the spacetime code is as follows:



Given that the channel coefficients remain constant over two consecutive symbol periods, the signals at the two receive antennas are given by a set of linear equations as follows:-

At time $t_{1}$,

$$
\begin{aligned}
& n_{1}=h_{1} s_{1}+h_{2} s_{2}+n_{1} \\
& n_{2}=-h_{1} s_{2}^{+}+h_{2} s_{1}^{+}+n_{2}
\end{aligned}
$$

And at time $\mathrm{t}_{1}+\mathrm{T}$,

$$
\begin{aligned}
& n_{y}-h_{y} s_{1}+h_{4} s_{2}+n_{d} \\
& n_{4}=-h_{7} s_{2}^{*}+h_{4} s_{1}^{*}+n_{4}
\end{aligned}
$$

where, $n_{1}, n_{2}, n_{2}$ and $n_{4}$ are complex samples of independent Gaussian distributed noise and interference. 


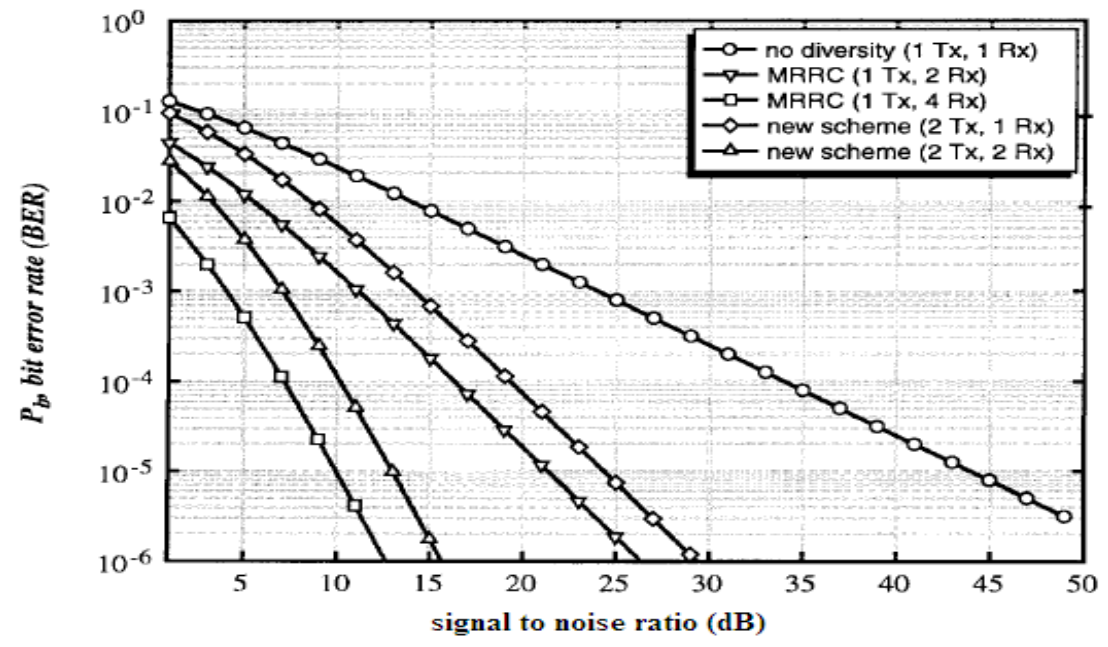

Figure 2. BER performance comparison of coherent BPSK with MRRC and two-branch transmit diversity in Rayleigh fading. (Source: Almouti, 1998)

The performance of communication systems is, to a large extent, dependent on the type and efficiency of modulation techniques employed. The published performance of Almouti 2x2 MIMO scheme (fig. 2) employed coherent BPSK which uses only real components of the signal constellation in the simulation [3]. If the simulation is carried out using QPSK where both real and imaginary components of the constellation are used, what will the BER performance be?

Quite a number of performance results have been reported for different antenna constellations, different environmental conditions, different modulation techniques and a lot more scenarios. For instance, [4] has reported that mutual coupling between antenna elements in a MIMO antenna constellation could be either positive or negative on the error performance of the Almouti scheme. [5] simulated the Almouti STBC in MPSK with increasing number of receiving antennas, and concluded that in Rayleigh fading channels, if the number of receiving antennas is kept constant the performance of STBC improves with increasing number of transmit antennas.

\section{SIMULATION OF 2X2 ALMOUTI MIMO SCHEME}

Various MIMO simulation results carried out using MATLAB or C++ with different parameters both in baseband and pass band have been reported in several papers. The methods used for the simulation are very diverse in the choice of the parameters for, say, modulation technique, signal detection, channel model etc. The simulations vary also as a result of the assumptions made in carrying them out.

After setting some parameters such length of symbols, modulation order, simulating the Almouti $2 \times 2$ starts with the transmit side where random data is generated as an input information to the system. This data is in the form of $1 \mathrm{~s}$ and 0 s randomly generated with equal probability of occurrence. This data is then split into two, giving rise to data1 and data2 which are fed to a baseband modulator (MPSK or MQAM) from where the symbols S1 and S2 are produced. These symbols are then encoded using the Almouti space-time coding scheme generating the code word $S$ given by 
International Journal of Information Sciences and Techniques (IJIST) Vol.2, No.5, September 2012

$$
\mathrm{s}=\left[\begin{array}{cc}
s_{1} & s_{2} \\
-S_{2} & S_{1}^{*}
\end{array}\right]
$$

It is usually necessary, when simulating any system, to have a reference with which to compare and confirm the veracity of the obtained results. As the simulation is for $2 \times 2$ system using the Almouti Space Time codes, slow fading Rayleigh channel was assumed in line with the assumptions of [3]. So the encoded Almouti code word is then passed through a simulated Rayleigh slow fading channel perturbed by complex Gaussian noise factors of zero mean and a variance of $\sigma^{2}$.

It is pertinent to note that, as the channels are randomly generated and assumed known at the receiver, channel estimation is not implemented in the simulation. The received signals which are affected by noise are combined together using equations (7) and (8) to produce outputs $\mathrm{C} 1$ and $\mathrm{C} 2$ which are then demodulated in the detector.

$C_{1}-h_{1}^{*} \eta_{1}+h_{2} n_{2}^{*}+h_{y}^{*} \eta_{4}+h_{4} r_{4}^{*}$

$C_{2}=h_{2}^{*} r_{1}-h_{1} t_{2}^{*}+h_{4}^{*} n_{3}-h_{2} r_{4}^{*}$

\section{SIMULATION RESULTS AND ANALYSIS}

The performance curves obtained with MPSK are shown in fig 3.

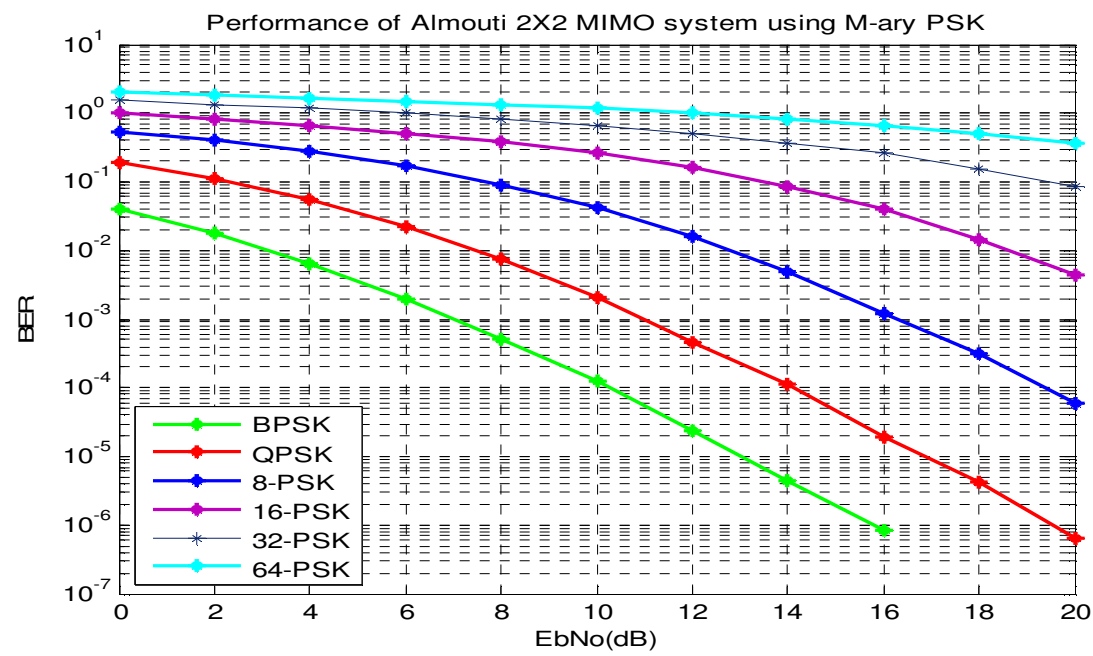

Figure 3. Performance curves of Almouti 2x2 MIMO scheme using M-ary PSK

The results show that as the modulation order increases more energy is needed to achieve a given error probability. Increase in the modulation order means an increase in the number of bits per symbol to be processed. For instance, it can be seen that for a BER of $10^{-2}$ almost additional $4 \mathrm{~dB}$ of energy is required for QPSK over what will be needed with BPSK. Generally we see from the curves in Figure 3 that for the same bit error rate of $10^{-2}$, going from $\mathrm{M}=4$ to $\mathrm{M}=8$ will require about $5.8 \mathrm{~dB}$, and from $\mathrm{M}=8$ to $\mathrm{M}=16$ about $6 \mathrm{~dB}$ to achieve the same performance. 


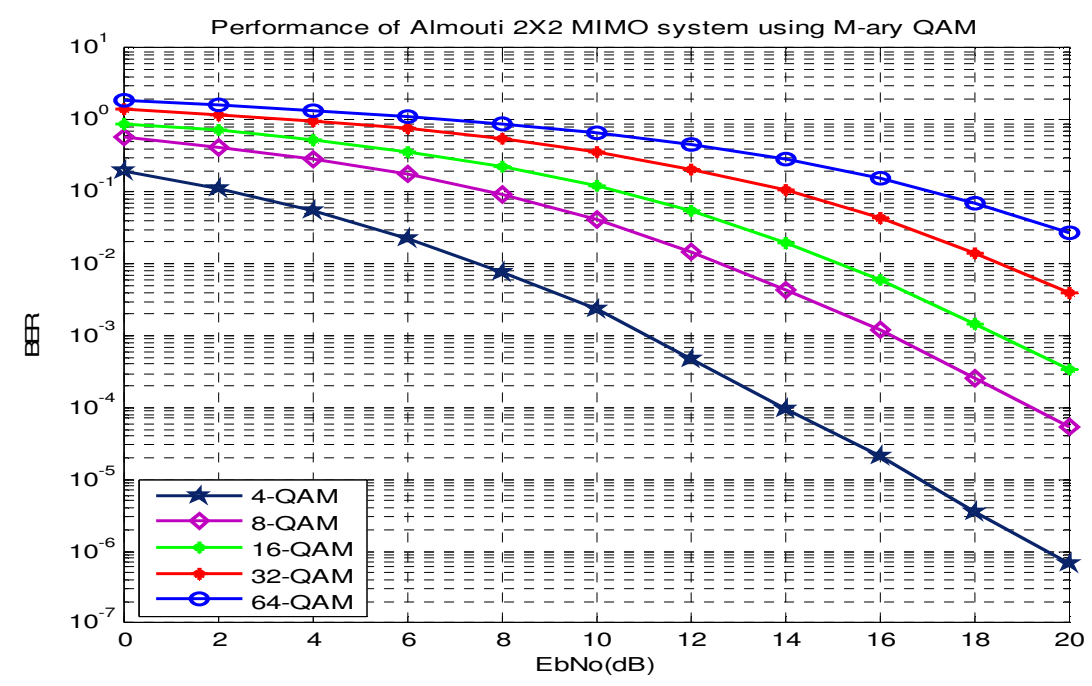

Figure 4. Performance of Almouti 2x2 MIMO system using M-ary QAM

The performance curves obtained with QAM are shown in fig 4. The curves obtained for the Mary QAM also show adherence to the expected behaviour for such modulation scheme. It can however be seen that in comparison to the M-ary PSK curves of fig. 3, while 4QAM is exactly the same as QPSK in performance, there is a distinct difference in the performance of the higher order schemes between both QAM and PSK generally. For instance, we see from fig.3 that, a BER of $10^{-3}$ is obtained at Eb/No of about $11 \mathrm{~dB}$ for QPSK which is the same for 4QAM in fig. 4. However, if we look at say 16PSK and 16QAM, we find that to achieve a BER of $10^{-2}$ will require about 3.4dB less when using 16QAM than what will be required for 16PSK (which is approximately $18.6 \mathrm{~dB}$ ).

Fig. 5 shows more closely the direct comparison between the performance of the simulated system using PSK and QAM with modulation order of 16. Here we see that 16QAM is more power efficient than 16PSK and this is attributable to the nature of the signal space diagrams of the two modulation schemes. Even though QAM and PSK both have 2 - dimensional signal spaces, the signal points, in the case of PSK, lie on a circle of fixed radius, $\sqrt{E_{s}}$, where $E_{z}$ is the energy per symbol. So, as the modulation order increases, the signal points tend to be more and more crowded together thereby increasing the probability of error. However, for QAM the signal points are distributed in the signal space without being confined to lie on a circle. 
International Journal of Information Sciences and Techniques (IJIST) Vol.2, No.5, September 2012

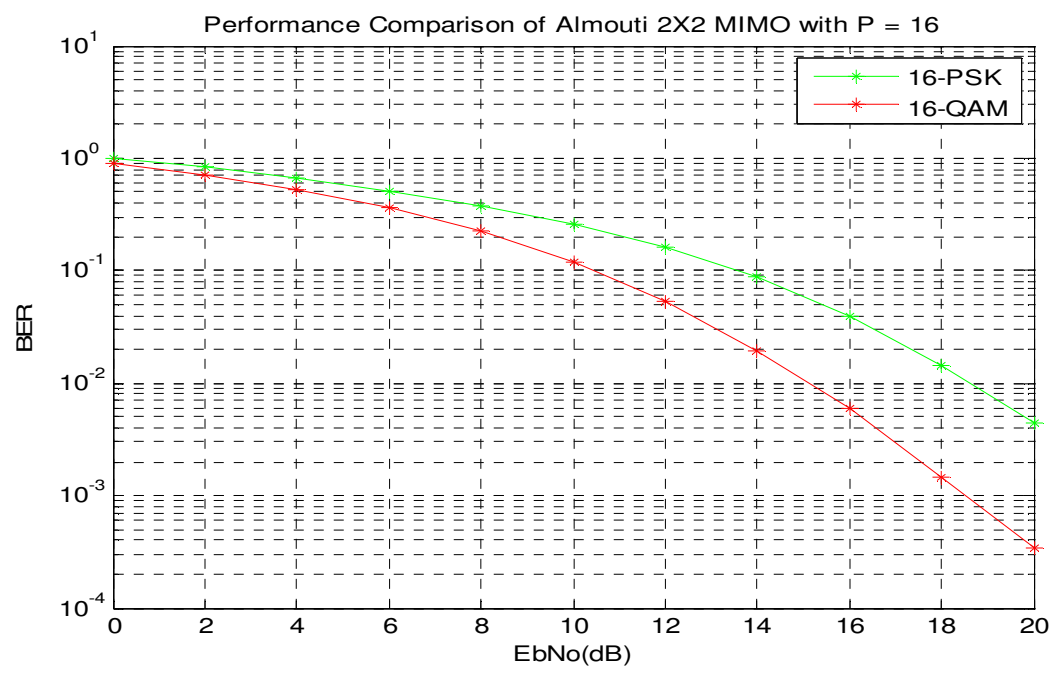

Figure 5. Performance comparison of Almouti 2x2 MIMO with 16-PSK and 16-QAM

While BPSK is better than QPSK and 16QAM (fig 6) in terms of power usage (i.e. lower EbNo for a given BER), its data rate is lower. In fig. 6 we see that 16QAM which is more susceptible to noise and interference due to its dependence on amplitude requires relatively more power for a given BER, but it should be noted that it has a higher spectral efficiency when compared with BPSK or QPSK [6].

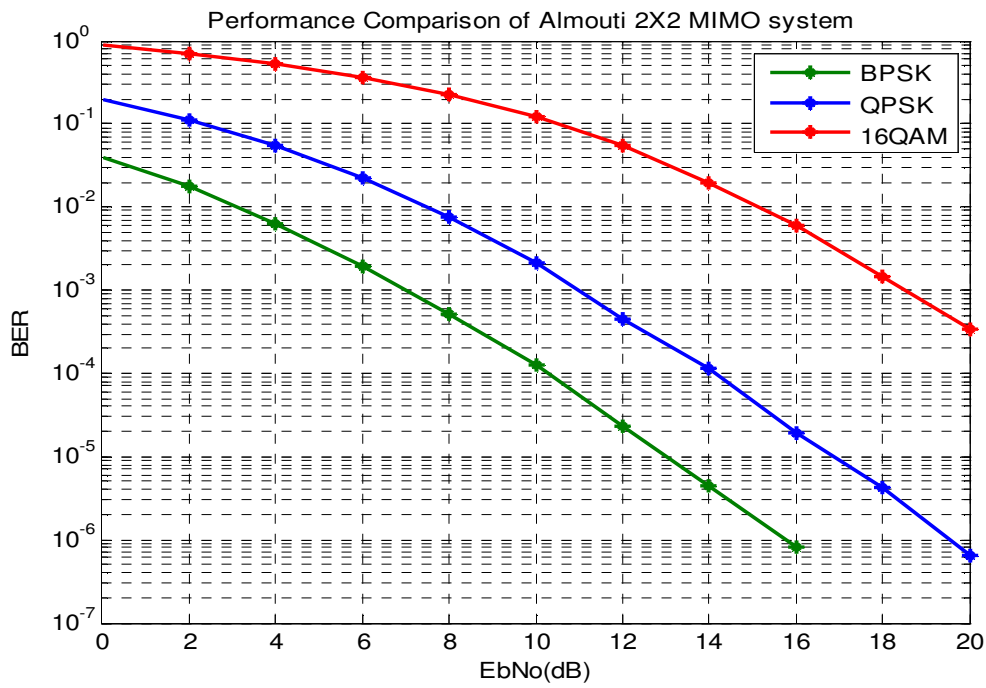

Figure 6. Performance comparison of Almouti 2x2 MIMO system with BPSK, QPSK and 16-QAM

We see from Fig. 7 that there is just about $1 \mathrm{~dB}$ additional energy required in the simulated result to achieve the same error performance as the theoretical curve. However, for the 16QAM and 64QAM, the energy required for a given error is much more as can be seen in Figs. 8 and 9. For a given error probability of $10^{-1}$, for instance, the energy required in the simulated curve shows the need for approximately $7 \mathrm{~dB}$ above the theoretical requirement in the case of 16QAM. But, with 
International Journal of Information Sciences and Techniques (IJIST) Vol.2, No.5, September 2012

64QAM, almost twice that amount of energy is necessary to achieve the same level of performance with the theoretical result.

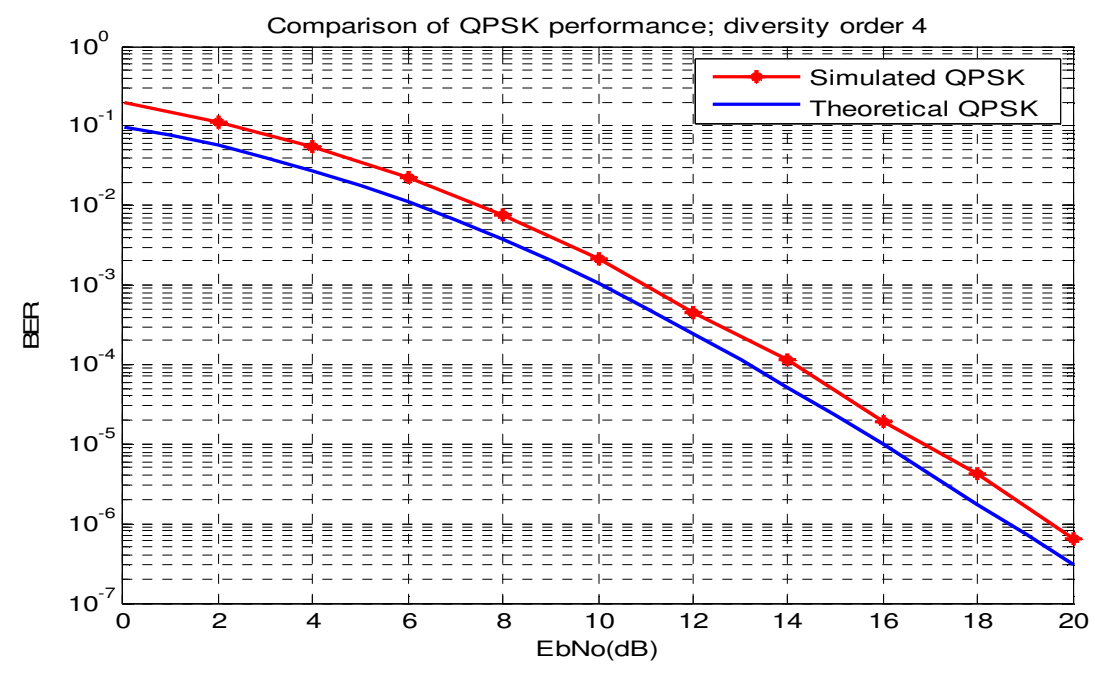

Figure 7. Performance of simulated vs theoretical MIMO scheme with diversity order 4 in Rayleigh slow fading (QPSK).



Figure 8. Performance of simulated vs theoretical MIMO scheme with diversity order 4 in Rayleigh slow fading (16QAM). 


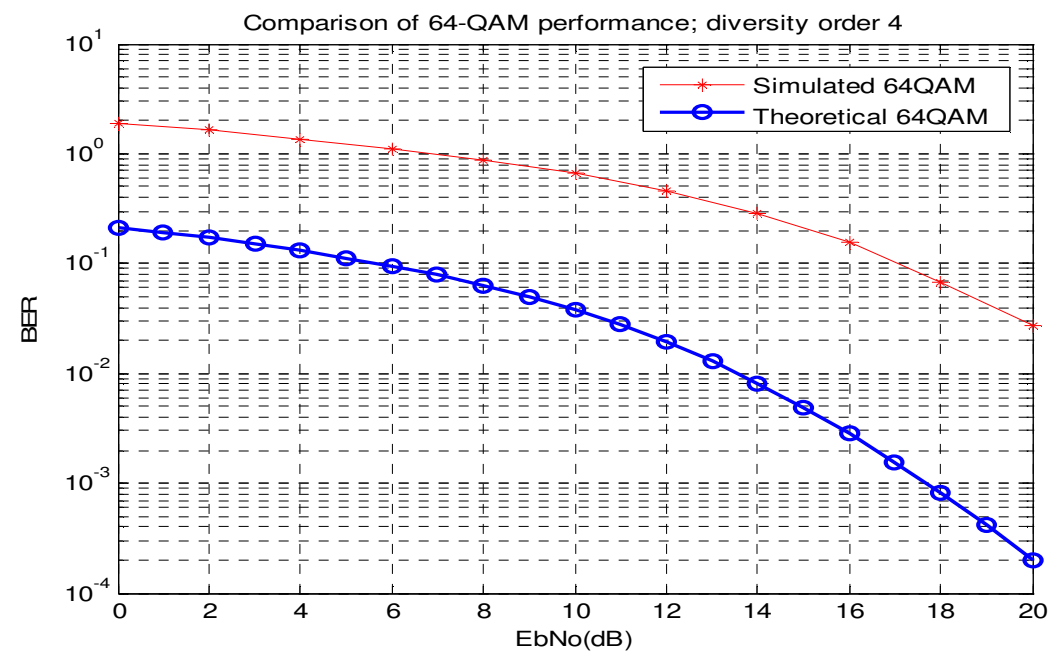

Figure 9. Performance of simulated vs theoretical MIMO scheme with diversity order 4 in Rayleigh slow fading (64QAM).

\section{CONCLUSION}

The Almouti 2x2 MIMO scheme was simulated using both M-ary PSK and M-ary QAM so as to be able to compare the performance. Using the BER tool on the MATLAB, theoretical performance curves for the two chosen modulation schemes were obtained and compared with simulated results. Basic assumptions were made in line with the published work of [3] in simulating the $2 \times 2$ system using PSK.. Generally, the curves obtained in the simulations follow the expected behaviour for such modulation schemes.

While we can conclude that QPSK outperforms 16QAM in error performance as could be seen in Fig.6, it is to be noted that QPSK gives only half the data rate possible with 16QAM. So there has to be a trade-off; get better error performance for less data rate or get more data rate at the expense of degraded error performance.

The 2x2 MIMO systems was simulated in Rayleigh slow fading and with channel perfectly assumed to be known at the receiver. Consequently, further simulation could be suggested where the channel is assumed to be unknown at the receiver, but to be extracted through the received signal. This will then involve channel estimation at the receiver, and furthermore maximum likelihood detection could be employed. These simulations could be done in different modulation schemes and different constellation orders.

For simulations with higher order modulations, the maximum likelihood decoder will be very complex if there are many antennas. And it was noted that one of the advantages of the Almouti scheme is its simple structure and linear processing at the receiver for two transmit antennas. Furthermore, it is not known yet whether for general complex constellations the Almouti spacetime code and the simple linear processing will give the maximum diversity gain when the number of transmit antennas is more than two and this could be another area for future research. 
International Journal of Information Sciences and Techniques (IJIST) Vol.2, No.5, September 2012

\section{REFERENCES}

[1] Tarokh V, Naguib A, Seshadri N and Calderbank,A. R, (1999)' Combined Array Processing and Space-Time Coding' IEEE Transactions on Information Theory, vol. 45, no. 4

[2] Gesbert D, Shafi M, Shiu D, Smith P J, and Naguib A, (2003)' From Theory to Practice: An Overview of MIMO Space-Time Coded Wireless Systems' IEEE Journal on Selected Areas in Communications, vol. 21, no. 3: 281-302.

[3] Almouti S. M. (1998) 'A Simple Transmit Diversity Technique for Wireless Communications' IEE Journal on Select Areas in Communications, vol. 16 no. 8: 1451-1458

[4] Abouda, A. A., El-Sallabi, H.M. and Häggman, S.G.,(2006) 'Effect of Mutual Coupling on BER Performance of Almouti Scheme,' Proc. of IEEE International Symposium on Antennas and Propagation, AP-S 2006, pp. 4797-4800, Jul. 2006, New Mexico, USA.

[5] Yuyu, L. and Yongzhi, L (2007) 'Research and Performance Analysis of Space-Time Block Codes in MIMO system' IEEE International Conference on Wireless Communications, Networking and Mobile Computing, 2007. WiCom 2007, pp. 118-121

[6] Pearson, J., (1992) 'Basic Communication Theory’ Prentice Hall International (UK) Ltd.

[7] Schindler, S. (2006) Introduction to MIMO Systems, Application Note 1MA102, Rhode\&Schwarz, http://www.mwjournal.cm/BGDownload/MIMO.pdf accessed: on 6th August 2008.

[8] Shafi, M., Gesbert, D., Shiu, D., Smith, P. J. and Tranter W. H. (2003) 'Guest Editorial MIMO Systems and Applications: Part I' IEEE Journal on Selected Areas in Communications, vol. 21, no. 3.

[9] Proakis J. G. (1995) Digital Communications, $3^{\text {rd }}$ Edition McGraw Hill Book Co., Singapore

[10] Calhoun G. M. (2003) Third Generation Wireless Systems Artech House, Inc., Norwood MA, USA.

[11] Almers P., Bonek E., Burr A., Czink N., Debbah M., Degli-Esposti V., Hofstetter H, Kyösti P, Laurenson D, Matz G. Molisch A F, Oestges C, and Özcelik H, (2007)' Survey of Channel and Radio Propagation Models for Wireless MIMO Systems' EURASIP Journal on Wireless Communications and Networking vol. 2007, Article ID 19070, p 19 doi:10.1155/2007/19070

[12] Abu-Rgheff M. A. (2007) Introduction to CDMA Wireless Communications Elsevier Ltd, Oxford, UK.

[13] J. Jialitto, M. Stege, M. Lohning, M. Bronzel and G. Fettweis, "A vector Channel Model with Stochastic Fading Simulation, In PIMRC'99, Osaka Japan, September 1999

[14] Ernst Borek, et al, "A Geometry-Based Stochastic MIMO Channel Model for 4G Indoor Broadband Packet Access", XXVIIIth General Assembly of International Union of Radio Science (URSI), New Delhi, India $23^{\text {rd }}$ October, 2005

\section{Authors}

A.S. Mindaudu obtained his diploma in Electrical/Electronic Engineering from Kaduna Polytechnic, BSc in Electrical/ Electronics Engineering from Ahmadu Bello University both in Nigeria. He then proceeded to University of Plymouth, UK and obtained an MSc in Communication Engineering. He was a Lecturer at the Kaduna Polytechnic before joining a telecommunication Company where he raised to the position of Deputy General Manager Network Planning and later Projects. He is presently pursuing his PhD at Usman Danfodio University, Nigeria.

A. M.Miyim obtained his diploma in Electronic/Communication Engineering from Kaduna Polytechnic, Nigeria and an MSC in Communication Engineering from Odessa National Academy of Telecommunications (ONAT), Ukraine. He was a Lecturer at the Kaduna Polytechnic and Abubakar Tafawa Balewa University bauchi(ATBU), Nigeria before joining a telecommunication Company where he handled the CDMA Network Expansion Project. He is presently pursuing his $\mathrm{PhD}$ at the Universiti Kebangsaan, (UKM), Malaysia. His interest is in Algorithm Development, Wireless Network and 4G. 